\title{
UNIVERSALLY TORSIONLESS AND TRACE MODULES
}

BY

\author{
GERALD S. GARFINKEL
}

ABSTRACT. We study, over an arbitrary ring $R$, a class of right modules intermediate between the projective and the flat content modules. Over the ring of rational integers these modules are the locally free abelian groups. Over any commutative ring they are the modules which remain torsionless under all scalar extensions. They each possess a certain separability property exactly when $R$ is left semihereditary.

We define $M$ to be universally torsionless if the natural map $M \otimes A \rightarrow$ $\operatorname{Hom}\left(M^{*}, A\right)$ is monic for all left modules $A$. We give various equivalent conditions for $M$ to be universally torsionless, one of which is that $M$ is a trace module, i.e. that $x \in M \cdot M^{*}(x)$ for all $x \in M$. We show the countably generated such modules are projective.

Chase showed that rings over which products of projective or flat modules are also, respectively, projective or flat have other interesting properties and that they are characterized by certain left ideal theoretical conditions. We show similar results hold when the trace or content properties are preserved by products.

1. Introduction. We call a right module $M$ universally torsionless (UTL) if the natural transformation $M \otimes A \rightarrow \operatorname{Hom}\left(M^{*}, A\right)$ is monic for all left modules $A$. In $\S 2$ we justify the notation by showing that over a commutative ring, $M$ is UTL exactly if it remains torsionless under all (or all commutative) extensions of the base ring.

Properties equivalent to UTL are enumerated in our basic Theorem 3.2. We show that UTL modules coincide with the trace modules of $\mathrm{Ohm}$ and Rush [11] and the flat, strict Mittag-Leffler modules of Raynaud and Gruson [8]. A projective-like characterization of a UTL module is that every homomorphism into $M$ with pure image is "finitely split." Thus every projective module is UTL. The converse holds for countably generated modules. The UTL property is preserved by arbitrary scalar extensions.

Presented to the Society, January 18, 1974 under the title Completely torsionless modules; received by the editors July 24, 1974.

AMS (MOS) subject classifications (1970). Primary 16A50; Secondary 13C10, 13F05, 16A14, $20 \mathrm{~K} 20$.

Key words and phrases. Torsionless module, projective module, flat module, locally free abelian group, trace module, content module, semiheditary ring, Mittag-Leffler module, pure submodule, locally projective module, coherent ring. 
$\S 4$ is devoted to studying two generalizations of locally tree abelian groups - the locally projective (LP) and the projectively separable (PS) modules. We show PS $\Rightarrow$ UTL $\Rightarrow$ LP in general and PS $=$ UTL (= LP) over a left semihereditary (and commutative) ring. Neither the LP nor the PS property is preserved by scalar extensions.

Given a module property $X$, we call a ring right $\Pi-X$ if each product of right modules with property $X$ also has property $X$. Chase [6] characterized the right $\Pi$-projective and the right $\Pi$-flat rings. In $\$ 5$ we study the right $\Pi$-trace rings. Characterizations of trace modules over such rings generalize well-known characterizations of locally free abelian groups. The left Noetherian condition is sufficient but not necessary for a ring to be right $\Pi$-trace. A necessary but probably not sufficent condition is that the ring be left coherent and satisfy the infinite intersection property on finitely generated left ideals. The latter condition is related to the content property studied by Ohm and Rush in [11].

$\$ 6$ deals with the relation between a collection of rings and their cartesian product. In particular we are interested in when such rings are П-projective, П-flat, П-trace or П-content.

Our notational conventions are as follows. All rings have an identity. The symbol $R$ always denotes a ring and unless otherwise specified, all modules $M$ are right unitary $R$-modules. Hom $=\operatorname{Hom}_{R^{\prime}} \otimes=\otimes_{R^{\prime}} M^{*}=\operatorname{Hom}(M, R)$ and map means $R$-homomorphism. We call $S$ a ring extension of $R$ if there is (an identity preserving) ring homomorphism from $R$ to $S$. $S$ is then considered a two sided $R$-module in the obvious manner. "Finitely generated" will be abbreviated to "f.g." Other notations and definitions will be introduced as needed.

I wish to take this opportunity to thank R. Keown, M. Orzech and F. Sandomierski for stimulating conversations about topics in this paper.

2. Torsionless modules. We start by formally stating two nonstandard conventions we will employ.

If $A$ is a two-sided $R$-module, we define $R\langle A\rangle$ to be the ring whose underlying abelian group is $R \times A$ and whose multiplication is given by

$$
\left(r_{1}, a_{1}\right)\left(r_{2}, a_{2}\right)=\left(r_{1} r_{2}, r_{1} a_{2}+a_{1} r_{2}\right), \quad r_{i} \in R, a_{i} \in A \text {. }
$$

$R\langle A\rangle$ is called the trivial ring extension of $R$ by $A$.

For any right $R$-module $M$ and left $R$-module $A$ let

$$
\Gamma=\Gamma_{R}(M, A): M \otimes A \rightarrow \operatorname{Hom}\left(M^{*}, A\right)
$$

be the unique map such that $\Gamma(m \otimes a)(u)=u(m) \cdot a$ for $m \in M, a \in A$ and $u \in M^{*}$. Bass calls $M$ torsionless if $\Gamma(M, R)$ is monic and reflexive if $\Gamma(M, R)$ is an isomorphism. 
Proposition 2.1. Let $M$ be a right $R$-module. Then $M \otimes S$ is $S$-torsionless for all ring extensions $S$ iff $\Gamma(M, A)$ is monomorphism for all two-sided $R$-modules $A$.

Proof. For each ring extension $S$ of $R$ consider the commutative diagram (2.1.1) where $\theta$ is induced by the natural isomorphism

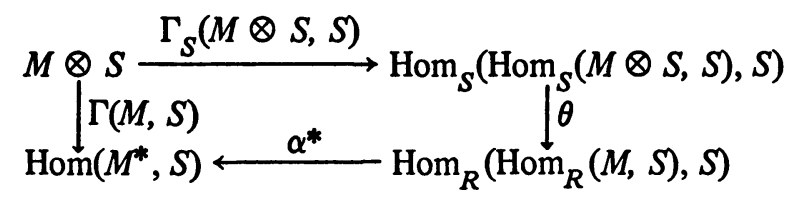

$\operatorname{Hom}_{R}(M, S) \cong \operatorname{Hom}_{S}(M \otimes S, S)$ and the inclusion $\operatorname{Hom}_{S} \subseteq \operatorname{Hom}_{R}$ and $\alpha$ : $\operatorname{Hom}(M, R) \rightarrow \operatorname{Hom}(M, S)$ is induced by $R \rightarrow S$.

Suppose $\Gamma(M, A)$ is monic for all two-sided $R$-modules $A$. Then if $S$ is any ring extension of $R, \Gamma_{S}(M \otimes S, S)$ is monic since it factors the monomorphism $\Gamma(M, S)$. Thus $M \otimes S$ is $S$-torsionless.

For any two-sided $R$-module $A$ let $S=R\langle A\rangle$. The decomposition $S=R \times$ $A$ yields

$$
\operatorname{Hom}(M, S)=\operatorname{Hom}(M, R) \times \operatorname{Hom}(M, A) .
$$

Thus $g=\theta \Gamma_{S}(M \otimes S, S)$ is defined by linearity and

$$
g[m \otimes(r, a)](u, w)=(u(m), w(m))(r, a)=(u(m) r, w(m) r+u(m) a)
$$

for $m \in M, r \in R, a \in A, u \in M^{*}, w \in \operatorname{Hom}(M, A)$. Thus for $x \in M \otimes A$,

$$
g(x)(u, w)=(0, \Gamma(x)(u)) .
$$

Note $\theta$ is always a monomorphism. Thus when $M \otimes S$ is $S$-torsionless, $g$ is monic on $M \otimes S$ and hence $\Gamma(M, A)$ is monic on $M \otimes A$.

We shall call $M$ universally torsionless (UTL) if $\Gamma(M, A)$ is monic for all left $R$-modules $A$ and universally ring torsionless (URTL) if $M \otimes S$ is $S$-torsionless for all ring extensions $S$ of $R$. In this notation, we have just shown UTL $\Rightarrow$ URTL.

THEOREM 2.2. For a module $M$ over a commutative ring $R$, the following are equivalent.

(1) $M$ is UTL.

(2) $M$ is URTL.

(3) $M \otimes S$ is $S$-torsionless for all commutative ring extensions $S$ of $R$.

Proof. For any module $A, R\langle A\rangle$ is commutative. Thus by the proof of Proposition 2.1 we have (3) $\Rightarrow(1)$. Clearly (1) $\Rightarrow(2)$ by Proposition 2.1 and (2) $\Rightarrow(3)$ is trivial. 
COROLLARY 1. Let $S$ be a commutative ring extension of the commutative ring $R$. If $M$ is UTL as an R-module, then $M \otimes S$ is UTL as an S-module.

Proof. If $T$ is a commutative ring extension of $S$, then $(M \otimes S) \otimes_{S} T$ $\cong M \otimes T$ is $T$-torsionless.

Let $A$ be the class of rings for which URTL = UTL. We have really proved Corollary 1 for rings in $A$. We shall show $A$ is a very large class, but we do not know if it includes all rings. (In $\S 3$ we will prove Corollary 1 for arbitrary rings by a different method.)

We call a submodule $P$ of $M$ pure if $P \otimes A \rightarrow M \otimes A$ is monic for all left modules $A$ and ideal pure if $P \cap M I=P I$ for all left ideals $I \subseteq R$. Various properties of purity are discussed in [4] and [12]. Those properties we will be concerned with are summarized below.

Lemma 2.3. If $P$ is a submodule of $M$, then (i) $\Rightarrow$ (ii) $\Rightarrow$ (iii).

(i) $M / P$ is flat.

(ii) $P$ is a pure submodule of $M$.

(iii) $P$ is an ideal pure submodule of $M$. If $M$ is flat, then (iii) $\Rightarrow$ (i) and $P$ is flat. If each f.g. left R-module is a direct sum of cyclics, then (iii) $\Rightarrow$ (ii).

Proof. For each left module $A$ consider the exact sequence

$$
\operatorname{Tor}_{1}(M, A) \rightarrow \text { Tor }_{1}(M / P, A) \rightarrow P \otimes A \rightarrow M \otimes A .
$$

If $M / P$ is flat, then $\operatorname{Tor}_{1}(M / P, A)=0$ and so $P \otimes A \rightarrow M \otimes A$ is monic.

Thus (i) $\Rightarrow$ (ii). Clearly (ii) $\Rightarrow$ (iii) since (iii) is equivalent to

(iii)' $P \otimes R / I \rightarrow M \otimes R / I$ is monic for all left ideals $I$.

If $M$ is flat, then (*) shows (ii) $\Rightarrow$ (i) and since $M$ and $M / P$ are flat the exact sequence $\operatorname{Tor}_{2}(M / P, A) \rightarrow \operatorname{Tor}_{1}(P, A) \rightarrow \operatorname{Tor}_{1}(M, A)$ shows $P$ is flat. Thus we need to show (iii) $\Rightarrow$ (ii) when $M$ is flat. But in this case, each exact sequence $0 \rightarrow A^{\prime} \rightarrow A \rightarrow A^{\prime \prime} \rightarrow 0$ of left modules induces a commutative exact diagram

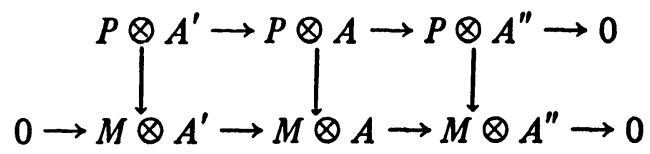

Hence if $P$ is ideal pure, one can easily show that $P \otimes A \rightarrow M \otimes A$ is monic for each f.g. left module $A$. But clearly the latter is equivalent to (ii). When (iii)' holds, $P \otimes A \rightarrow M \otimes A$ is monic for direct sums of cyclic left modules $A$, thus (iii) $\Rightarrow$ (i) if each f.g. left module is such a direct sum.

COROLlary 2. If $R$ contains a central subring $K$ as a $K$-pure submodule, then URTL $=$ UTL. 
Proof. For each $M_{R}$ and ${ }_{R} A, M \otimes A \rightarrow M \otimes A \otimes_{K} R$ is monic. Hence $\Gamma(M, A)$ is monic if $\Gamma\left(M, A \otimes_{K} R\right)$ is monic. Since $A \otimes_{K} R$ is a twosided $R$-module, we see by Proposition 2.1 that $M$ is URTL iff $M$ is UTL.

COROLLARY 3. If $(R,+)$ is either torsion free or torsion, then URTL $=$ UTL over $R$.

Proof. If $(R,+)$ is torsion free, let

$$
K=Z_{*}=\{r \in R: n r=m 1 \text { for some } n \neq 0, m \in Z\} .
$$

Since $R$ is torsion-free, $K$ is a central subring of $R$ which is a localization of $\mathbf{Z}$ (the rational integers). Note $K$ is a PID and is an ideal pure $K$-submodule of $R$. Thus by Lemma 2.3 and Corollary 2, URTL $=$ UTL over $R$.

If $(R,+)$ is torsion, let $K \cong \mathrm{Z} /(n)$ be the base ring of $R$. By Lemma 2.3 and Corollary 2, we need only show $K \cap m R=m K$ for all $m \in \mathbf{Z}$. Suppose $m r \in K$. Let $d=\operatorname{gcd}(m, n)$ with $m=m_{1} d$ and $n=n_{1} d$. Then $n_{1} m r=n m_{1} r=$ 0 . Hence $m r=d a$ for some $a \in K$. Since $\operatorname{gcd}\left(m_{1}, n\right)=1$, there is $b \in K$ with $a=m_{1} b$. Thus $m r=m b \in m K$. (Actually $K$ is thus even a $K$-direct summand of $R$ by [7, p. 11].)

Proposition 2.4. For $M_{R}$ the following are equivalent:

(1) $M$ is UTL.

(2) $M$ is URTL and flat.

Proof. (1) $\Rightarrow$ (2). Suppose $\alpha:{ }_{R} A \rightarrow{ }_{R} B$ is monic. Consider the commutative diagram

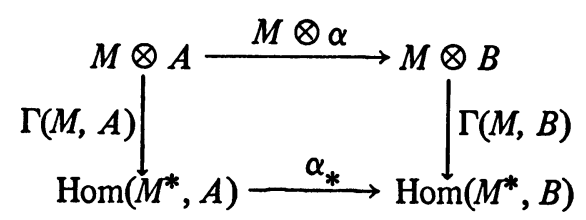

Since $\alpha_{*}$ and $\Gamma(M, A)$ are monic, so is $M \otimes \alpha$. Thus $M$ is flat. By Proposition 2.1, $M$ is URTL.

(2) $\Rightarrow(1)$. For any ${ }_{R} A$ let $B=A \otimes_{\mathrm{Z}} R$ and define $\alpha:{ }_{R} A \rightarrow{ }_{R} B$ by $\alpha(a)=$ $a \otimes 1$. The map $\beta: B \rightarrow A$ defined by $\beta(a \otimes r)=r a$ (and linearity) is an abelian group splitting of $\alpha$. Hence $\alpha$ and (since $M$ is flat) $M \otimes \alpha$ are monic. $B$ is a two-sided $R$-module, so by Proposition $2.1 \Gamma(M, B)$ is monic. Thus the commutativity of (*) shows that $\Gamma(M, A)$ is monic. Thus $M$ is UTL.

3. Basic properties. We now investigate how UTL modules interact with other left and right modules.

For $\beta: G_{R} \rightarrow M_{R}$ and $n$ a natural number let $G(n)$ be $G(n): \forall x_{1}, \ldots, x_{n}$ $\in G, \quad{ }^{\exists} \varphi: M_{R} \rightarrow G_{R}$ with $\beta \varphi \beta\left(x_{i}\right)=\beta\left(x_{i}\right)$ for $i \leqslant n$. 
Lemma 3.1. For each $n, G(1) \Leftrightarrow \mathcal{G}(n)$.

Proof. By induction we need only show $\mathcal{G}(n) \Rightarrow \mathcal{G}(n+1)$. Suppose $G(n)$ is true and $x_{1}, \ldots, x_{n+1} \in G$. Since $G(n) \Rightarrow G(1)$, there is $\varphi_{1}: M \rightarrow G$ with $\beta \varphi_{1} \beta\left(x_{n+1}\right)=\beta\left(x_{n+1}\right)$. Let $y_{i}=x_{i}-\varphi_{1} \beta\left(x_{i}\right)$. By $\Theta(n)$ there is $\varphi_{2}: M \rightarrow$ $G$ with $\beta \varphi_{2} \beta\left(y_{i}\right)=\beta\left(y_{i}\right)$ for $i \leqslant n$. An easy calculation shows $\varphi=\varphi_{1}+\varphi_{2}-$ $\varphi_{2} \beta \varphi_{1}$ satisfies $\mathcal{G}(n+1)$.

We call a map $\beta: G_{R} \rightarrow M_{R}$ split if there is $\varphi: M_{R} \rightarrow G_{R}$ with $\beta \varphi \beta=\beta$, finitely split if it satisfies $G(1)$ and (ideal) pure if its image is (ideal) pure. Note finitely split $=$ split if the image of $\beta$ is finitely generated.

THEOREM 3.2. The following conditions are equivalent for any right $R$-module $M$.

(i) $M$ is UTL.

(ii) $M$ is a (ideal) pure submodule of a right UTL module $P$.

(iii) $\Gamma(M, A)$ is monic for each cyclic left $R$-module $A$.

(iv) Each $m \in M$ is a member of $M \cdot M^{*}(m)$ where $M^{*}(m)=\left\{u(m): u \in M^{*}\right\}$.

(v) For each $m \in M$, there are $m_{1}, \ldots, m_{n} \in M$ and $u_{1}, \ldots, u_{n} \in M^{*}$ with $m=\Sigma m_{i} u_{i}(m)$.

(vi) Each ideal pure map $\beta: G \rightarrow M$ finitely splits.

(vii) Each epimorphism $\beta: G \rightarrow M$ finitely splits.

(viii) For each f.g. submodule $N$ of $M$ there is a f.g. free right module $F$ and maps $\alpha: F \rightarrow M$ and $\theta: M \rightarrow F$ with $\alpha \theta$ the identity on $N$.

(ix) For each f.g. submodule $N$ of $M$ there are $m_{1}, \ldots, m_{n} \in M$ and $u_{1}, \ldots, u_{n} \in M^{*}$ with $x=\Sigma m_{i} u_{i}(x)$ for $x \in N$.

(x) For each epimorphism $\beta: G \rightarrow M$ the induced map $\operatorname{Hom}(C, \beta)$ is epic whenever $C$ is f.g. and for each $m \in M$ there is an $\alpha \in \operatorname{End}(M)$ with $\alpha(m)=$ $m$ and the image of $\alpha$ is contained in a f.g. submodule of $M$.

Proof. We show the first nine conditions imply each other cyclicly and then show (ix) $\Rightarrow(x) \Rightarrow$ (vii). We omit the trivial verifications (i) $\Rightarrow$ (ii), (iv) $\Rightarrow$ (v) and (vi) $\Rightarrow$ (vii).

(ii) $\Rightarrow$ (iii). For each cyclic left module $A$ consider the commutative diagram

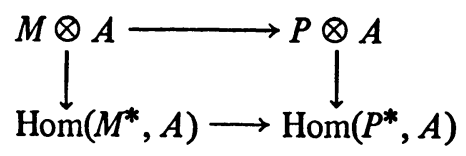

The upper map is monic since $M$ is pure in $P$ and the right-hand map is monic since $P$ is UTL. Thus the left map $\Gamma(M, A)$ is monic.

(iii) $\Rightarrow$ (iv). For $m \in M$ let $I=M^{*}(m)$ and let $f$ be the composition of the natural isomorphism $M / M I \rightarrow M \otimes R / I$ with $\Gamma(M, R / I)$. Clearly for $x \in M$ and 
$u \in M^{*}, f(x+M I)(u)=u(x)+I$ and thus $f(m+M I)=0$. However if (iii) holds then $f$ is monic and so $m \in M I$.

(v) $\Rightarrow$ (vi). Let $\beta: G \rightarrow M$ be ideal pure and $x \in G$. By (v) there are $m_{1}, \ldots, m_{n} \in M$ and $u_{1}, \ldots, u_{n} \in M^{*}$ with $\beta(x)=\Sigma m_{i} u_{i}(\beta x)$. Since the image of $\beta$ is ideal pure in $M$, there are $x_{1}, \ldots, x_{n} \in G$ with $\beta(x)=$ $\Sigma \beta\left(x_{i}\right) u_{i} \beta(x)$. Define $\varphi: M \rightarrow G$ by $\varphi(y)=\Sigma x_{i} u_{i}(y)$. Clearly $\beta \varphi \beta(x)=\beta(x)$ and so (vi) holds.

(vii) $\Rightarrow$ (viii). Suppose $\beta: G \rightarrow M$ an epimorphism with $G$ free. For each f.g. submodule $N$ of $M$, there is a f.g. submodule $H$ of $G$ with $\beta(H)=N$. Thus there is a map $\varphi: M \rightarrow G$ with $\beta \varphi \beta=\beta$ on $H$. Let $F$ be any f.g. free direct summand of $G$ containing $\varphi(N)$. Let $\alpha: F \rightarrow M$ be the restriction of $\beta$ to $F$ and let $\theta: M \rightarrow F$ be the composition of $\varphi$ with the projection onto $F$. It is then trivial to verify that $\alpha \theta$ is the identity on $N$.

(viii) $\Rightarrow$ (ix). If $N$ is a f.g. submodule of $M$ let $F, \alpha$ and $\theta$ be as in (viii). Let $y_{1}, \ldots, y_{n}$ be a basis of $F$. Then $y=\Sigma y_{i} v_{i}(y)$ for each $y \in F$ where the $v_{i}$ 's are the coordinate functions. Hence for $x \in N$,

$$
x=\alpha \theta(x)=\alpha \sum y_{i} v_{i} \theta(x)=\sum m_{i} u_{i}(x)
$$

where $m_{i}=\alpha\left(y_{i}\right)$ and $u_{i}=v_{i} \theta$.

(ix) $\Rightarrow$ (i). Let $A$ be a left module and $x=\Sigma x_{j} \otimes a_{j}$ where $x_{1}, \ldots, x_{r} \in$ $M$ and $a_{1}, \ldots, a_{r} \in A$. Let $N=\Sigma x_{j} R$ and choose $m_{1}, \ldots, m_{n} \in M$ and $u_{1}, \ldots, u_{n} \in M^{*}$ with $y=\Sigma m_{i} u_{i}(y)$ for $y \in N$. Letting $\Gamma=\Gamma(M, A)$ we see

$$
x=\sum m_{i} u_{i}\left(x_{j}\right) \otimes a_{j}=\sum m_{i} \otimes \Gamma(x)\left(u_{i}\right)
$$

and hence $x=0$ if $\Gamma(x)=0$. Thus $\Gamma(M, A)$ is monic for each $A$ and so $M$ is UTL.

(ix) $\Rightarrow(\mathrm{x})$. Suppose $\beta: G \rightarrow M$ is epic and $f \in \operatorname{Hom}(C, M)$ with $C$ f.g. Then $f(C)$ is f.g. and hence there are $m_{1}, \ldots, m_{n} \in M$ and $u_{1}, \ldots, u_{n} \in M^{*}$ with $f(c)=\Sigma m_{i} u_{i} f(c)$ for $c \in C$. Let $g: C \rightarrow G$ be defined by $g(c)=\Sigma y_{i} u_{i} f(c)$ where $\beta\left(y_{i}\right)=m_{i}$. It is easy to see that $\beta g=f$ and thus $\operatorname{Hom}(C, G) \rightarrow \operatorname{Hom}(C, M)$ is onto. For $m \in M$, we can choose $m_{1}, \ldots, m_{n} \in M, u_{1}, \ldots, u_{n} \in M^{*}$ with $m=\Sigma m_{i} u_{i}(m)$. Let $\alpha: M \rightarrow M$ be defined by $\alpha(x)=\Sigma m_{i} u_{i}(x)$. Clearly $\alpha$ has the desired properties and hence $(\mathrm{x})$ is true.

(x) $\Rightarrow$ (vii). Suppose $\beta: G \rightarrow M$ is epic and $m \in M$. By (x), we can choose $\alpha \in \operatorname{End}(M)$ with $\alpha(m)=m$ and $\alpha(M) \subseteq N$ f.g. Again by (x) we can find $g: N \rightarrow G$ such that $\beta g: N \longrightarrow M$ is the inclusion map. Let $\varphi=g \alpha$. Then

$$
m=\alpha(m)=\beta g(\alpha(m))=\beta \varphi(m)
$$

and the proof of the theorem is completed. 
The first consequence we derive from the theorem is the promised strengthening of Corollary 1 to Theorem 2.2.

COROLLARY 1. For any UTL right $R$-module $M$ and any ring extension $S$ of $R$ the right $S$-module $M \otimes S$ is $U T L$.

Proof. We show condition (v) is satisfied for each $x=\Sigma x_{j} \otimes s_{j}$ in $M \otimes$ $S$. By condition (ix) there are $m_{i} \in M$ and $u_{i} \in M^{*}$ with each $x_{j}=\Sigma m_{i} u_{i}\left(x_{j}\right)$. Then each

$$
\begin{gathered}
u_{i} \otimes S \in \operatorname{Hom}_{S}(M \otimes S, S) \text { and } \\
x=\sum_{m_{i}} \otimes u_{i}\left(x_{j}\right) s_{j}=\sum\left(m_{i} \otimes 1\right) \cdot\left(u_{i} \otimes S\right)(x) .
\end{gathered}
$$

The next two corollaries are immediate consequences of (i) $\Leftrightarrow$ (vii).

COROLLARY 2. Every projective module is UTL.

COROLlaRY 3. Every f.g. UTL module is projective.

COROLLARY 4. Every f.g. pure submodule of a UTL module is a projective direct summand.

Proof. Use the previous corollary, part (vi) of the theorem and the remark about finitely split maps.

COROLLARY 5. Every countably generated submodule of a UTL module $M$ is contained in a countably generated pure projective submodule.

Proof. Given a sequence $\left\{y_{n}\right\}$ in the module $M$, by condition (ix) and induction we can find for each $n$ finitely many $x_{n, j} \in M, u_{n, j} \in M^{*}$ such that if $\varphi_{n}(x)=\Sigma x_{n, j} u_{n, j}(x)$ then $\varphi_{n}$ is the identity on $y_{n}$ and on $x_{n-1, j}$. Clearly $\Sigma x_{n, j} R$ contains each $y_{n}$, is ideal-pure and thus pure and has $\left\{x_{n, j} ; u_{n, j}\left(1-\varphi_{n-1}\right)\right\}$ as a projective basis $[5, \mathrm{p} .132]$.

If $R$ is a commutative ring, a module $M$ satisfying condition (iv) of Theorem 3.2 is called a trace module by Ohm and Rush in [11] and is called a flat strict Mittag-Leffler module by Gruson and Raynaud. (See for example Proposition 2.3.4 on p. 76 of [8] .) Ohm and Rush also used commutative ring techniques to prove Corollaries 2 and 3 above.

We favor the Ohm and Rush notation and shall hereafter use the term "trace module" in place of "UTL module." However, since "trace" is not a true adjective, we shall continue our previous usage in phrases such as " $M$ is UTL."

Corollary 4 indicates that when $R=\mathbf{Z}$ (the ring of rational integers) trace modules may be related to the locally free abelian groups. Indeed in the next section we shall show that when $R=\mathbf{Z}$ the two concepts coincide. 
Corollary 5 is reminiscent of Kaplansky's result [9] that every projective module is the direct sum of countably generated modules. The natural question is: Does Kaplansky's result remain true when "trace module" is substituted for "projective module"? The answer is NO-the result is already false when $R=\mathbf{Z}$. By Corollary 5 the direct sums of countably generated trace $Z$-modules are the free abelian groups but by our previous remark the trace $\mathbf{Z}$-modules form the class of locally free abelian groups. $\mathbf{Z}^{\mathbf{Z}}$ for example was shown by Reinhold Baer [2] to be locally free but not free abelian. (In $\S \S 5$ and 6 we will follow the example of Chase in [6] and explore the consequences of $R^{R}$ being a trace module over $R$.)

4. Locally free abelian groups. In this section we study two generalizations of locally free abelian groups. One property is stronger and one is weaker than the trace property. All three coincide when the ring is commutative and semihereditary.

We start by defining our first generalization of locally free abelian groups as follows. For any right module $M$ over an arbitrary ring $R$ let $\mu_{R}(M)$ be the minimal number of elements needed to generate $M$ and let the rank of $M$ (denoted $\operatorname{rank}_{R}(M)$ be the minimum $\mu_{R}(N)$ where $N$ is a submodule of $M$ and $N \otimes S \rightarrow M \otimes S$ is epic for some ring $S$ containing $R$. Finally we call $M$ locally projective (LP) if each pure submodule of finite rank is a f.g. projective direct summand.

REMARKS. (i) If $R$ is a domain with quotient field $k$ then $\operatorname{rank}_{R}(M)=$ $\operatorname{dim}_{k}(M \otimes k)$.

Proof. Clearly $\operatorname{rank}(M) \leqslant \operatorname{dim}(M \otimes k)$. Suppose rank $M=\mu(N)$ and $N \otimes S \rightarrow M \otimes S$ is epic with $R \subseteq S$. If $M \otimes k=(N \otimes k) \oplus P$ then $M \otimes k \otimes$ $S=N \otimes k \otimes S \Rightarrow P \otimes S=0$. But $P$ is $R$-flat and thus $P \subseteq P \otimes S$. Hence $\operatorname{dim}(M \otimes k)=\operatorname{dim}(N \otimes k) \leqslant \mu(N)=\operatorname{rank}(M)$.

(ii) Over the ring of integers our definition of $L P$ modules coincides with the standard definition of locally free abelian groups.

Proposition 4.1. Every trace module $M$ is $L P$.

Proof. Let $P$ be a pure submodule of $M$ with rank $P=\mu(N)<\infty$ where $N \otimes S \rightarrow P \otimes S$ is epic and $R \subseteq S$. By Theorem 3.2(vi) there is a map $\varphi: M$ $\rightarrow P$ which is the identity on $N$. By parts (ii) and (ix) of Theorem 3.2 there are $u_{1}, \ldots, u_{n} \in P^{*}$ and $x_{1}, \ldots, x_{n} \in P$ such that $\theta$ is the identity on $N$ where $\theta: P \rightarrow P$ is defined by $\theta(x)=\Sigma x_{i} u_{i}(x)$. We need only show that the restriction $f$ of $\theta \varphi$ to $P$ is the identity. Since $f$ is the identity on $N$ and $N \otimes S$ $\rightarrow P \otimes S$ is epic we have $f \otimes S$ is the identity on $P \otimes S$. However, $P$ is flat and thus $P \subseteq P \otimes S$. Hence $f$ is the identity on $P$.

By Theorem 3.2 it is easily seen that a module is UTL if each element is 
contained in a projective (or even UTL) direct summand. Thus an LP module is UTL if each element is contained in a pure submodule of finite rank. We will show this occurs when $R$ is commutative and semihereditary.

Suppose $R$ is a commutative ring. A nonzero divisor in $R$ is called regular. The total quotient ring of $R$ is its localization at the set of regular elements. If $N$ is a submodule of $M$, we define the purification of $N$ as

$$
N_{*}=\{x \in M: x \lambda \in N \text {, some regular } \lambda \in R\} .
$$

(If $R$ is semihereditary $N_{*}$ is the smallest pure submodule containing $N$. In general $N_{*}$ is not pure in $M$.)

PROPOSITION 4.2. If $R$ is a commutative semihereditary ring, then each $L P$ module $M$ is a trace module.

Proof. We show $N_{*}$ is a pure submodule of finite rank when $N$ is f.g. It is well known (and easily seen) that the total quotient ring $k$ is absolutely flat. Thus $(M \otimes k) /(N \otimes k)$ is $k$-flat and hence $R$-flat. Since $R$ is semihereditary, submodules of flat modules are flat. See $[6, \mathrm{p} .468]$. Thus $M / N_{*}$ is flat since $N_{*}=$ $M \cap(N \otimes k)$. Hence $N_{*}$ is pure. Clearly rank $N_{*}=\operatorname{rank} N$.

We next give a counterexample which shows that UTL $\neq$ LP and the LP property is not preserved by a change of scalars. Since the argument is complicated we break it up into a lemma and a proposition. Recall a Prüfer domain is a semihereditary integral domain.

LEMMA 4.3. Suppose $R$ is a domain and for each $n \geqslant 0$ we are given a free right $R$-module $F_{n}$ of rank $n$, an $R$-map $\theta_{n}: F_{n} \rightarrow F_{n+1}$ and an ideal $I_{n}$ satisfying

(i) $\theta_{n}$ is monic.

(ii) $\theta_{n}\left(F_{n}\right) \subseteq F_{n+1} I_{n+1}$.

(iii) $\bigcap_{p} I_{n+1} \ldots I_{n+p}=0$ for $n=0,1,2, \ldots$.

(iv) $\left(F_{n+1} \cdot x\right) \cap \theta_{n}\left(F_{n}\right)=\theta_{n}\left(F_{n} \cdot x\right)$ for $x \in R$.

Then $M=\lim _{n} F_{n}$ is a nonzero LP module with $M^{*}=0$. Suppose $S$ is a Prüfer domain containing $R$ such that $\bigcap_{p}\left(I_{n+1} \ldots I_{n+p} S\right)=0$. Then $M \otimes S$ is not $L P$ as an $S$-module.

Proof. Let $M_{n}$ be the image of $F_{n}$ in $M$. By (i) $M_{n} \cong F_{n}$ and hence $M$ is nonzero. Let $I_{n+1} \ldots I_{n+p}=I_{n, p}$. By (ii) $M_{n} \subseteq \bigcap M I_{n, p}$. Thus for $u \in$ $M^{*}, u\left(M_{n}\right) \subseteq \bigcap I_{n, p}=0$. Since $M=\lim M_{n}$ we thus see $M^{*}=0$.

We demonstrate that $M$ is an LP module by showing that the only pure submodule $P$ of finite rank is the zero module. Clearly any such $P$ must be the purification of a f.g. submodule $N$ which in turn must be contained in some $M_{n}$. By (iv) we see that $M_{n}$ is its own purification and thus contains $P$. Hence, for each $p \geqslant 1, P=P \cap M_{n} \subseteq P \cap M I_{n, p}=P I_{n, p}$. Thus $P$ is a submodule of $\bigcap_{p} M_{n} I_{n, p}$ which is zero since $M_{n}$ is free. 
Note that the first three conditions on $M$ forced $M^{*}=0, M \neq 0$ and thus $M$ non UTL. Hence we need to show $M \otimes S$ satisfies the corresponding three conditions when we replace $F_{n}, \theta_{n}$ and $I_{n}$ by corresponding objects over $S$.

Since tensor products commute with direct limits we see $M \otimes S \cong$ $\lim _{\rightarrow}\left(F_{n} \otimes S\right)$. Suppose $B_{n}$ is the matrix representing $\theta_{n}$. Since $\theta_{n}$ is monic, the rank of $B_{n}$ is $n$ over the quotient field of $R$ and thus over the quotient field of $S$. Hence $\theta_{n} \otimes S$ is monic. Condition (ii) is obvious and condition (iii) is the intersection hypothesis on $S$.

Proposition 4.4. Let $k$ be a field and $R=k\left[x_{1}, \ldots, x_{n}, \ldots\right]-$ the polynomial ring in a countable number of variables. Let $I_{n}$ be the ideal generated by $x_{1}, \ldots, x_{n}$; let $F_{n}$ be the standard module of row vectors and let $\theta_{n}: F_{n} \rightarrow$ $F_{n+1}$ be multiplication by the matrix

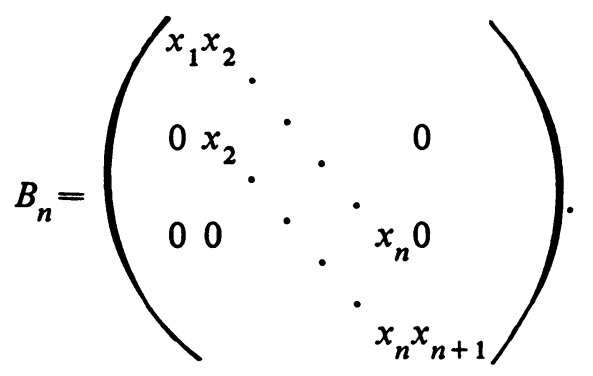

Finally let $T=k\left(\ldots, x_{i} / x_{j}, \ldots\right)$ and $S=T\left[x_{1}\right]$. Then $R, I_{n}, F_{n}, \theta_{n}$ and $S$ satisfy the hypotheses and thus conclusions of Lemma 4.3.

Proof. Conditions (i), (ii) and (iii) are obvious. Condition (iv) states that, for $c_{1}, \ldots, c_{n+1}, d_{1}, \ldots, d_{n}, x \in R,\left(c_{1}, \ldots, c_{n+1}\right) x=\left(d_{1}, \ldots, d_{n}\right) B_{n}$ implies $x \mid d_{i}$ for $i \leqslant n$. Since $R$ is a UFD it clearly suffices to prove condition (iv) when $x$ is a prime element. If $x$ does not divide each $d_{i}$, let $m$ be the minimal integer such that $x \chi d_{m}$. Let $d_{0}=0$. Then $x \mid d_{i}$ for $i<m$ and $c_{m} x=\left(d_{m-1}+d_{m}\right) x_{m}$. Since $x$ is prime, $x \mid x_{m}$. Thus for $\left.i>m, x\right\rangle x_{i}$ (since $x_{i}$ and $x_{m}$ are relatively prime) and therefore $x \mid d_{n}$ and $x \mid\left(d_{i-1}+d_{i}\right)$ for $m<i \leqslant n$. But $d_{m}=$ $\left(d_{m}+d_{m+1}\right)-\left(d_{m+1}+d_{m+2}\right)+\ldots \pm d_{n}$ and hence $x \mid d_{m}$ also.

As for $S$, it is clearly a PID and thus Prüfer. The intersection property holds since $I_{n} \cdot S=S x_{1}$ for each $n$.

We now study the second generalization of locally free abelian groups.

Suppose $M$ is a left or a right module. If $N$ is a submodule of $M$ we let $N^{\prime}=\left\{u \in M^{*}: u(N)=0\right\}$. Letting $\Gamma: M \rightarrow M^{* *}$ be the canonical map we set $\bar{N}=\Gamma^{-1}\left(N^{\prime \prime}\right)$. We call $\bar{N}$ the closure of $N$ and call $N$ closed if $N=\bar{N}$. We say $M$ is projectively separable (PS) if the closure of each f.g. submodule is a f.g. projective direct summand of $M$.

We omit the proof of the following facts. 
Lemma 4.5. Let $N$ and $P$ be submodules of $M$.

(i) $N^{\prime}=\bar{N}^{\prime}$.

(ii) $\bar{N}$ is closed.

(iii) If $N \subseteq P$, then $\bar{N} \subseteq \bar{P}$.

(iv) If $M$ is torsionless then any direct summand is closed.

The statement of the following proposition is derived from [3].

Proposition 4.6. Suppose $N=x_{1} R+\ldots+x_{n} R$ is a submodule of the right $R$-module $M$. Define $\varphi: M^{*} \rightarrow R^{n}$ by $u \mapsto\left(u\left(x_{1}\right), \ldots, u\left(x_{n}\right)\right)$. Let $\theta$ be the corestriction of $\varphi$ onto its image $S \subseteq R^{n}$ and let $\Gamma: M \rightarrow M^{* *}$ be the canonical map.

(i) $N^{\prime}=\operatorname{Ker} \theta$ and $S$ is isomorphic to the image of the restriction map $M^{*} \rightarrow \bar{N}^{*}$.

(ii) $\theta^{*}\left(S^{*}\right)=N^{\prime \prime}$ and there is a commutative diagram

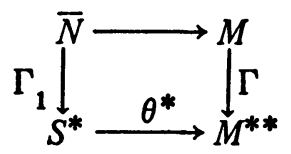

(iii) If $M$ is reflexive or UTL, $\Gamma_{1}$ is an isomorphism.

(iv) If $\Gamma_{1}$ is an isomorphism and $\theta: M^{*} \rightarrow S$ splits, then $\bar{N}$ is a direct summand of $M$.

(v) If $\bar{N}$ is a direct summand of $M$, then $\theta: M^{*} \rightarrow S$ splits and $S \cong \bar{N}^{*}$.

Proof. (i). Trivial.

(ii). The exact sequence $0 \rightarrow N^{\prime} \stackrel{i}{\rightarrow} M^{*} \rightarrow S \rightarrow 0$ induces the exact sequence $0 \longrightarrow S^{*} \stackrel{\theta^{*}}{\longrightarrow} M^{* *} \stackrel{i^{*}}{\longrightarrow} N^{*}$. Hence $\theta^{*}\left(S^{*}\right)=\operatorname{Ker} i^{*}=N^{\prime \prime}$. Also $\Gamma^{-1} \theta^{*}(S)=\bar{N}$ and so there is a map $\Gamma_{1}$ rendering the diagram commutative.

(iii). $\Gamma_{1}$ is monic since $\Gamma$ is. By (ii) we need only show that $N^{\prime \prime} \subseteq \Gamma(M)$. This is certainly true if $M$ is reflexive. Assume $M$ is UTL and $\alpha \in N^{\prime \prime}$. We wish to find $x \in M$ with $\alpha(u)=u(x)$ for all $u \in M^{*}$. Since $M$ is UTL, we can find $u_{1}, \ldots, u_{r} \in M^{*}$ and $m_{1}, \ldots, m_{r} \in M$ with $y=\Sigma m_{i} u_{i}(y)$ for $y \in N$. Thus for any $u \in M^{*}, u-\Sigma u\left(m_{i}\right) u_{i}$ restricts to the zero map on $N$ and is thus in $N^{\prime}$. Hence it is in $\operatorname{Ker} \alpha$ and so $\alpha(u)=\Sigma u\left(m_{i}\right) \alpha\left(u_{i}\right)=u\left(\Sigma m_{i} \alpha\left(u_{i}\right)\right)$.

(iv). Suppose $g: S \rightarrow M^{*}$ is a splitting for $\theta$ and $\Gamma_{1}$ is an isomorphism. It is then easy to check that $\Gamma_{1}^{-1} g^{*} \Gamma$ is a projection of $M$ onto $\bar{N}$.

(v). $\theta$ splits since its kernel $N^{\prime}=\bar{N}^{\prime}$ is a direct summand of $M^{*}$. Clearly the restriction map $M^{*} \rightarrow \bar{N}^{*}$ is epic and so $S \cong \bar{N}^{*}$.

COROLLARY. If $R$ is left Noetherian and left hereditary, then each reflexive right $R$-module is $P S$.

Proof. Suppose $N$ is a f.g. submodule of the reflexive module $M$. As- 
suming the notation of Proposition 4.6 we note that $S$ is a f.g. projective left module since it lies in a f.g. free left module. Since $\Gamma_{1}: \bar{N} \rightarrow S^{*}$ is an isomorphism, $\bar{N}$ is a f.g. projective right module. By part (iv) of Proposition 4.6, $\bar{N}$ is a direct summand.

LEMmA 4.7. For a left ideal $I$ of an arbitrary ring $R$, the following are equivalent:

(i) $I=M^{*}(x)$ where $x$ is in the f.g. free right module $M$.

(ii) $I=M^{*}(x)$ where $x$ is in the right trace module $M$.

(iii) $I$ is $f . g$.

Proof. (i) $\Rightarrow$ (ii). Trivial.

(ii) $\Rightarrow$ (iii). By Theorem 3.2, $x=\Sigma m_{i} u_{i}(x)$ for $m_{i} \in M, u_{i} \in M^{*}$. It is easily verified that the $u_{i}(x)$ generate $I$.

(iii) $\Rightarrow$ (i). If $I=R a_{1}+\ldots+R a_{n}$ let $x=\left(a_{1}, \ldots, a_{n}\right)$. It is easily checked that $I=\left(R^{n}\right)^{*}(x)$.

Proposition 4.8. A right module $M$ is PS iff $M$ is UTL and $M^{*}(x)$ is projective for each $x$ in $M$.

Proof. $(\Rightarrow)$. Suppose $M$ is PS. Each f.g. submodule $N$ of $M$ satisfies condition (ix) of Theorem 3.2 since $\bar{N}$ does. Thus $M$ is UTL. For any $x=x_{1}$ in $M$, let $N=x R$ and $S=M^{*}(x)$. Since $\bar{N}$ is a direct summand, $S \cong \bar{N}^{*}$ by (v) of Proposition 4.6. Since $\bar{N}$ is a f.g. projective module so is $M^{*}(x)=S$.

$\Leftrightarrow)$. Suppose $M$ is UTL with $M^{*}(x)$ projective for each $x \in M$. We need to show $\bar{N}$ is a f.g. projective direct summand whenever $N$ is a f.g. submodule. We shall induct on $\mu(N)=$ the minimal number of generators for $N$.

In case $\mu(N)=1$, let $N=x R$ and $S=M^{*}(x)$. Since $M$ is UTL, part (iii) of Proposition 4.6 shows that $\Gamma_{1}: \bar{N} \rightarrow S^{*}$ is an isomorphism. Thus since $S$ is projective by our hypothesis and f.g. by the last lemma, $\bar{N}$ is f.g. and projective. Also since $S$ is projective, $\theta: M^{*} \rightarrow S$ splits and so by (iv) of Proposition $4.6, \bar{N}$ is a direct summand.

For the inductive step we may assume $N=P+x R$ where $\bar{P} \oplus L=M$. Let $x=y+z$ where $y \in L$ and $z \in \bar{P}$ and let $Q=P+y R$. It is easy to check that $N^{\prime}=Q^{\prime}$ and hence $\bar{N}=\bar{Q}$. Thus we may assume $N=Q$, i.e. that $x \in L$. Thus $\bar{N}=\bar{P} \oplus A$ where $A$ is the closure of $x R$ in $L$. Thus we need only show $A$ is a f.g. projective direct summand of $L$. But $L$ has the same properties as $M$, i.e. $L$ is UTL and $L^{*}(x)$ is projective for $x \in L$. Thus we are reduced to the case $N=x R$ which is the case $\mu(N)=1$.

A consequence of the method of proof of Proposition 4.8 is the following.

CoRollary 1. A right module $M$ is $P S$ if the closure of each cyclic module is a f.g. projective direct summand. 
Corollary 2. A domain $R$ is $P S$ as a module over itself.

Proof. $R$ is the closure of each of its nonzero submodules.

The following theorem is a generalization of results of Kaplansky [9], Albrecht [1] and Bass [3].

THEOREM 4.9. The following statements are equivalent for any ring $R$.

(i) $R$ is left semihereditary.

(ii) Every right trace $R$-module is $P S$.

(iii) Every f.g. free right $R$-module is $P S$.

Proof. (ii) $\Rightarrow$ (iii) is trivial. (iii) $\Rightarrow$ (i) $\Rightarrow$ (ii) by Proposition 4.8 and Lemma 4.7.

COROLlARY 1. The PS property is not preserved by a change of scalars.

Proof. Let $R$ by any non left semihereditary ring. By Theorem 4.9, $R$ possesses a free right module $F$ of finite rank which is not PS. Let $G$ be the free abelian group of rank that of $F$. Then $G$ is free over $\mathbf{Z}$ and thus PS over $\mathbf{Z}$, but $F \cong G \otimes_{\mathrm{Z}} R$ is not PS over $R$.

COROLLARY 2. The PS property is not preserved by finite direct sums.

Proof. Let $R$ be a non-Prüfer integral domain. By Corollary 2 to Proposition 4.8, $R$ is PS as a right $R$-module. By Theorem 4.9 some finite number of copies of $R$ is a non PS module.

COROLLARY 3. Over $\mathrm{Z}$ the LP, PS and trace modules all coincide with the locally free abelian groups.

5. Direct products of modules. In this section we study the preservation of the universally torsionless property by direct sums and products. Since the projective and flat properties are preserved by direct sums the following result is the expected one.

Proposition 5.1. A right module $M=\bigoplus M_{i}$ is UTL iff each $M_{i}$ is UTL.

Proof. $(\Leftrightarrow) \Gamma(M, A): M \otimes A \rightarrow \operatorname{Hom}\left(M^{*}, A\right)$ is additive in $M$. Thus if $\Gamma(M, A)$ is always monic, each $\Gamma\left(M_{i}, A\right)$ is always monic.

$\Leftrightarrow)$. Express each $x \in M$ as a finite sum $x=\Sigma x_{i}$ where $x_{i} \in M_{i}$. If $M_{i}$ is UTL then by Theorem $3.2, x_{i} \in M_{i} \cdot M_{i}^{*}\left(x_{i}\right)$. Clearly $M_{i}^{*}\left(x_{i}\right) \subseteq M^{*}(x)$. Thus $x \in M \cdot M^{*}(x)$ and hence by Theorem $3.2, M$ is UTL.

The situation with respect to the UTL property being preserved by direct products is much more complicated. We start our study of direct products by making the notational convention of writing $x=\Pi x_{\alpha}$ if $x \in \Pi M_{\alpha}$ and $x_{\alpha}$ is the $\alpha$-component of $x$. 
Lemma 5.2. $A$ left ideal $I$ of $R$ is f.g. iff $\left(\Pi M_{\alpha}\right) \cdot I=\Pi\left(M_{\alpha} \cdot I\right)$ for each collection $\left\{M_{\alpha}\right\}$ of right $R$-modules.

Proof. $\Leftrightarrow)$. Suppose $I$ is generated by $a_{1}, \ldots, a_{n}$ and $x \in \Pi\left(M_{\alpha} \cdot I\right)$ with $x=\Pi x_{\alpha}$. Then each $x_{\alpha}=\Sigma m_{\alpha, i} a_{i}$ for some $m_{\alpha, i} \in M$. Let $m_{i}=\Pi m_{\alpha, i}$. Clearly $x=\Sigma m_{i} a_{i}$ is in $\left(\Pi M_{\alpha}\right) \cdot I$. Thus $\Pi\left(M_{\alpha} \cdot I\right) \subseteq\left(\Pi M_{\alpha}\right) \cdot I$. The opposite inclusion is trivial.

$\Leftrightarrow)$. Let $x=\Pi x_{\alpha}$ where each $x_{\alpha}=\alpha$ for $\alpha \in I$. Then $x \in(R \cdot I)^{I}$ and hence $x \in R^{I} \cdot I$. Let $x=x_{1} a_{1}+\ldots+x_{n} a_{n}$ with $x_{i} \in R^{I}$ and $a_{i} \in I$. If $x_{i}=$ $\Pi x_{i, \alpha}$ then $\alpha=x_{\alpha}=\Sigma x_{i, \alpha} a_{i}$ and so $\left\{a_{1}, \ldots, a_{n}\right\}$ generates $I$.

For each right $R$-module $M$, we denote by $\Lambda=\Lambda(M): M \rightarrow R^{M^{*}}$ the canonical map defined by $\Lambda(m)(u)=u(m)$ for $m \in M, u \in M^{*}$. Note $\Lambda(M)$ factors thru $\Gamma: M \rightarrow M^{* *}$ and $M$ is torsionless iff $\Lambda$ is monic. Recall $f: A \rightarrow B$ is pure if the image of $f$ is pure in $B$.

Proposition 5.3. If $M$ is UTL, then $\Lambda: M \rightarrow R^{M^{*}}$ is a pure monomorphism.

Proof. For any left module $A$, the mapping $\Lambda \otimes A$ is monic since it factors the monomorphism $M \otimes A \rightarrow \operatorname{Hom}\left(M^{*}, A\right) \rightarrow A^{M^{*}}$.

THEOREM 5.4. For any ring $R$, the following are equivalent.

(i) A right module $M=\Pi M_{\alpha}$ is UTL if each $M_{\alpha}$ is UTL.

(ii) For any set $A$, the right module $R^{A}$ is UTL.

(iii) If $A$ is a set and $x \in M=R^{A}$, then $M^{*}(x)$ is f.g.

Proof. (i) $\Rightarrow$ (ii). Obvious.

(ii) $\Rightarrow$ (iii). By Lemma 4.7.

(iii) $\Rightarrow$ (ii). It suffices to show $x \in M \cdot M^{*}(x)$ whenever $x \in M=R^{A}$. If $u_{\alpha}: R^{A} \rightarrow R$ is the $\alpha$-component map, then $x=\Pi u_{\alpha}(x)$. Hence $x \in$ $\Pi\left(R \cdot M^{*}(x)\right)=(\Pi R) \cdot M^{*}(x)=M \cdot M^{*}(x)$ where the first equality follows from Lemma 5.2.

(ii) $\Rightarrow$ (i). By Proposition 5.3 each $M_{\alpha}$ is pure in $R^{M_{\alpha}^{*}}$. If $A$ is the disjoint union of the $M_{\alpha}^{*}$ 's we see that $M$ is ideal pure in $R^{A}$, which by (ii) is UTL. Hence by Theorem 3.2, $M$ is UTL.

We will call a ring $R$ right $\Pi-T$ if it satisfies the conditions of Theorem 5.4.

COROllary. $R$ is right $\Pi$ II-T if it is left Noetherian.

We next show that the right $\Pi-T$ rings are exactly those for which the converse of Proposition 5.3 is valid.

Proposition 5.5. For any ring $R$, the following are equivalent.

(i) $R$ is right $\Pi-T$.

(ii) A right R-module $M$ is UTL iff it is isomorphic to a pure submodule of $R^{A}$ for some set $A$. 
(iii) $A$ right $R$-module $M$ is UTL iff $\Lambda: M \rightarrow R^{*}$ is a pure monomorphism.

Proof. (i) $\Rightarrow$ (ii). By Proposition 5.3 and Theorems 5.4 and 3.2.

(ii) $\Rightarrow$ (iii). By Proposition 5.3.

(iii) $\Rightarrow$ (i). Since $\Lambda$ is always a split monomorphism when $M=R^{A}$.

LEMMA 5.6. If $N$ is a left module over the left semihereditary ring $R$, then $N^{*}$ is ideal pure in $R^{N}$.

Proof. Suppose $I$ is a left ideal and $u \in N^{*} \cap R^{N} I$ has the form $u=$ $\Sigma f_{i} a_{i}$ where $f_{i} \in R^{N}, a_{i} \in I$. Then $J=\Sigma R a_{i}$ is f.g. and thus projective and UTL. Hence there are $\varphi_{j} \in J^{*}, b_{j} \in J$ with $a=\Sigma \varphi_{i}(a) b_{i}$ for $a \in J$. For $x \in N, u(x)=$ $\Sigma f_{i}(x) a_{i} \in J$. Hence $u(x)=\Sigma \varphi_{i} u(x) \cdot b_{i}$. Thus $u=\Sigma \varphi_{i} u \cdot b_{i} \in N^{*} \cdot I$.

Corollary. Suppose $R$ is right $\Pi-T$ and left semihereditary. Then $N^{*}$ is right UTL for each left module $N$ and a right module $M$ is UTL iff it is torsionless and $M^{* *} / M$ is flat.

Proof. $N^{*}$ is UTL since it is a pure submodule of $R^{N}$. By Proposition 5.3, $M$ is UTL iff $\Lambda: M \rightarrow R^{M^{*}}$ is a pure monomorphism. Since $M^{* *}$ is pure in $R^{M^{*}}, \Lambda$ is a pure monomorphism iff $\Gamma: M \rightarrow M^{* *}$ is. $M^{* *}$ is UTL and thus flat. Hence $\Gamma$ is a pure monomorphism iff $M$ is torsionless and $M^{* *} / M$ is flat.

REMARK. Over the ring of rational integers Proposition 5.4 and the above corollary yield well-known characterizations of locally free abelian groups.

Note that the corollaries to Propositions 4.6 and 5.5 each give sufficient conditions for a right module over a left semihereditary ring $R$ to be UTL. However the weaker result needs the hypothesis that $R$ is left Noetherian while the stronger result needs the nominally weaker hypothesis that $R$ is right $\Pi-T$. At this point the only rings we know to be right $\Pi-T$ are the left Noetherian ones. In the next section we will give examples of commutative $\Pi-T$ rings which are non-Noetherian. In the rest of this section we will give some ideal theoretical properties of right $\Pi-T$ rings which show that many well-known rings are not right $\Pi-T$.

Recall we write $x=\Pi x_{i}$ if $x \in \Pi M_{i}$ with $x_{i}$ the $i$ th coordinate of $x$. For $x=\Pi x_{i} \in R^{A}$ we let $D(x)$ be the left ideal generated by the $x_{i}$ 's. Note $R^{A}=F^{*}$ where $F$ is a free left $R$ module and $D(x)=x(F)$.

LEMma 5.7. Suppose $x \in M=R^{A}$.

(1) $D(x) \subseteq M^{*}(x)$.

(2) If $D(x) \subseteq N^{*}(y)$ where $y$ is in the right module $N$, then $M^{*}(x) \subseteq N^{*}(y)$.

(3) If $y \in N=R^{B}$ and $D(y)=D(x)$, then $N^{*}(y)=M^{*}(x)$.

Proof. (1) For $\alpha \in A$, let $u_{\alpha}: R^{A} \rightarrow R$ be the $\alpha$-coordinate map. Then $x=\Pi u_{\alpha}(x)$ and $D(x)=\Sigma R u_{\alpha}(x) \subseteq M^{*}(x)$. 
(2) Suppose $u_{\alpha}(x)=f_{\alpha}(y)$ with $f_{\alpha} \in N^{*}$ for each $\alpha \in A$. Define $f: N \rightarrow$ $R^{A}$ by $f(z)=\Pi f_{\alpha}(z)$ for $z \in N$. Then $f(y)=x$ and hence for $u \in M^{*}, u(x)=$ $u f(y) \in N^{*}(y)$.

(3) Follows from (1) and (2).

Lemma 5.7 shows that for $x \in M=R^{A}, M^{*}(x)$ does not depend on $x$ or on $A$ but only on the ideal $D(x)$. Thus for any left ideal $I$ of $R$ we can define the left ideal $\hat{I}$ to be $M^{*}(x)$ where $x \in R^{A}$ is any point with $D(x)=I$. $\hat{I} \subseteq \hat{J}$.

LEMMA 5.8. Suppose $I$ and $J$ are left ideals of $R$ with $I \subseteq J$. Then $I \subseteq$

Proof. Suppose $I=D(x)$ and $J=D(y)$ with $x \in M=R^{A}$ and $y \in N=$ $R^{B}$. Then $I \subseteq \hat{I}$ and $J \subseteq \hat{J}$ by (i) of Lemma 5.7. Since $I \subseteq \hat{J}$, part (ii) of Lemma 5.7 shows $\hat{I} \subseteq \hat{J}$.

Proposition 5.9. Let $I$ be a left ideal of $R$. Then $I=\hat{I}$ if any of the following conditions hold:

(i) $I$ is f.g.

(ii) $I=0$ : $S=\{\lambda \in R: \lambda s=0 \forall s \in S\}$ where $S$ is any subset of $R$.

(iii) $I=\bigcap \hat{J}_{i}$ where each $J_{i}$ is a left ideal.

(iv) $I=\bigcap J_{i}$ where each $J_{i}$ is a f.g. left ideal.

Proof. (i). Use Lemma 4.7.

(ii). Let $I=0: S$ and $x \in M=R^{A}$ with $D(x)=I$. If $x=\Pi x_{\alpha}$ then each $x_{\alpha} \cdot S=0$. Hence for $s \in S, x s=0$. Thus for $u \in M^{*}, 0=u(x s)=u(x) s$. Therefore $\hat{I}=M^{*}(x) \subseteq 0: S=I \subseteq \hat{I}$.

(iii). Use Lemma 5.8.

(iv). Use (i) and (iii).

We shall call a ring $R$, right $\Pi-F$ (or right $\Pi-P$ ) if $M=\Pi M_{\alpha}$ is a flat (respectively projective) right $R$-module iff each $M_{\alpha}$ is.

Chase has given the following characterizations of these rings.

THEOREM 5.10 (Theorems 2.1 and 2.2 in [6]). For any ring $R$ the following are equivalent:

(i) $R$ is right $\Pi-F$.

(ii) $R^{A}$ is a flat right $R$-module for each set $A$.

(iii) $R$ is left coherent, i.e. each f.g. left ideal of $R$ is finitely presented.

(iv) 0 : $\{s\}$ is f.g. for each $s \in R$ and $I \cap J$ is f.g. if $I$ and $J$ are each f.g. left ideals of $R$.

THEOREM 5.11 (Theorem 3.3 of [6]). For any ring $R$ the following are equivalent:

(i) $R$ is right $\Pi-P$. 
(ii) $R^{A}$ is a projective right $R$-module for each set $A$.

(iii) $R$ is left coherent and $R$ satisfies the descending chain condition on principal left ideals.

If in the hypothesis of Chase's Theorem 3.1 in [6], we replace his concept of "pure" with our "ideal pure", then a close scrutiny of his proof shows we can replace "principal ideal" by "f.g. ideals" in his conclusion. This observation leads to the following modification of Theorem 5.11.

THEOREM 5.12. A ring is right П-P iff it is left coherent and it satisfies the descending chain condition on f.g. left ideals.

EXAMPLE 5.1. A commutative ring which satisfies d.c.c. on f.g. ideals but which is not coherent. Let $k$ be a field and $V$ an infinite dimensional vector space over $k$ and let $R=k\langle V\rangle$, the trivial ring extension of $k$ by $V . R$ satisfies d.c.c. on f.g. ideals since $V$ satisfies d.c.c. on f.g. subspaces. If $0 \neq v \in V$ then $0:\{v\}=V$ is a non f.g. ideal and thus by Theorem $5.10, R$ is not coherent.

Corollary. A left Noetherian ring is left Artinian iff it is right П-P.

We will say that $R$ satisfies the (finite) intersection property on f.g. left ideals if the intersection of any (finite) collection of f.g. left ideals is f.g. Note that $R$ satisfies the (finite) intersection property on f.g. left ideals if it is (respectively right $\Pi-F$ ) right $\Pi-P$.

THEOREM 5.13. If $R$ is right $\Pi-T$, then $R$ is left coherent and satisfies the intersection property on f.g. left ideals.

Proof. $R$ is left coherent since it is right $\Pi-F$. If $I=\bigcap J_{i}$ where each $J_{i}$ is a f.g. left ideal, then $I=\hat{I}$ by Proposition 5.5. Thus $I$ is f.g. by Lemma 4.7.

Theorem 5.13 shows that various well-known coherent rings are not $\Pi-T$.

EXAMPLE 5.2. If $k$ is a Noetherian commutative ring, then $R=$ $k\left[X_{1}, X_{2}, \ldots\right]$ is $\Pi-F$ but not $\Pi-T$.

PROOF. To show $R$ is coherent we note first that if an ideal is generated by $f_{1}, \ldots, f_{m}$, there is an $n \geqslant 0$ with each $f_{i} \in k\left[X_{1}, \ldots, X_{n}\right]=S$. Letting $I^{\prime}=\Sigma S f_{i}$, we note that $I^{\prime}$ has a finite $S$-presentation since $S$ is Noetherian. Since $R$ is free and thus flat over $S$, the finite $S$-presentation for $I^{\prime}$ can be lifted to a finite $R$-presentation for $R \otimes_{S} I^{\prime}$. But again the flatness of $R$ over $S$ yields $I \cong R \otimes_{S} I^{\prime}$.

To show $R$ is not ח-T we display a decreasing sequence of f.g. ideals whose intersection is not f.g.

$$
\begin{aligned}
& I_{1}=\left(X_{1}\right), \\
& I_{2}=\left(X_{1}^{2}, X_{1} X_{2}\right),
\end{aligned}
$$




$$
I_{n}=\left(X_{1}^{2}, X_{1} X_{2}^{2}, \ldots, X_{1} X_{2} \ldots X_{n-1}^{2}, X_{1} X_{2} \ldots X_{n-1} X_{n}\right) \text {. }
$$

Clearly $I=\bigcap_{n}$ is generated by all monomials of the form $X_{1} X_{2} \ldots X_{n-1} X_{n}^{2}$ and cannot be generated by any set of polynomials involving only a finite number of the indeterminates.

It is easy to see that every left semihereditary ring is left coherent and thus right $\Pi-F$. But even these rings need not be $\Pi-T$.

EXAmple 5.3. Let $R$ be a valuation ring with value group $G$. Since every f.g. ideal is free (on one generator) $R$ is coherent. However, it is easy to see that $R$ satisfies the intersection property on f.g. (principal) ideals iff $G$ is complete as a lattice.

The statement of Theorem 5.13 gives rise to two questions. (1) Is the intersection property on f.g. left ideals equivalent to some module property being preserved by direct products, i.e. is it $\Pi-X$ for some property $X$ ? (2) Is the converse of Theorem 5.13 true?

The answer to (1) is Yes and the property that is preserved is that of being a "content module." We do not know the answer to question (2). There is some strong evidence pointing to a negative answer and some weak evidence pointing to an affirmative answer. The negative evidence is that Theorem 5.13 is a consequence of "trace $\Rightarrow$ flat + content" whose converse is false. The positive evidence is the similarities in Theorems 6.7 and 6.8 .

If $x$ is a member of the right module $M$, we define

$$
C(x)=\mathcal{C}(x, M)=\bigcap\{I: x \in M I, I \text { a left ideal }\} .
$$

$C(x)$ is called the content or the content ideal of $x . M$ is called a content module if $x \in M C(x)$ for all $x \in M$. Content modules and ideals are studied by Ohm and Rush in [10] and [11]. Some of their properties are summarized below.

Proposition 5.14. Suppose $M$ is a right module and $x \in M$. Then

(1) $M^{*}(x) \subseteq C(x)$.

(2) $C(x)=\bigcap\{I: x \in M I$, I a f.g. left ideal $\}$.

(3) $x \in M C(x) \Rightarrow C(x)$ is f.g.

(4) $M$ is a trace module $\Rightarrow M$ is a content module.

(5) An ideal pure submodule of a content module is a content module.

(6) $M$ is a content module iff $M\left(\bigcap_{i}\right)=\bigcap\left(M I_{i}\right)$ for each collection of left ideals $\left\{I_{i}\right\}$.

Proof. See p. 51 of [11].

THEOREM 5.15. For any ring $R$, the following are equivalent.

(1) A right module $M=\Pi M_{\alpha}$ is a content module iff each $M_{\alpha}$ is a content module. 
(2) For any set $A, R^{A}$ is a right content module.

(3) For any set $A, C(x)$ is f.g. for each $x$ in the right module $R^{A}$.

(4) $I=\bigcap_{I_{i}}$ is f.g. if each $I_{i}$ is a f.g. left ideal of $R$.

Proof. (1) $\Rightarrow$ (2). Trivial.

(2) $\Rightarrow(3)$. A simple consequence of (3) in Proposition 5.14.

(3) $\Rightarrow$ (4). Let $\left\{x_{\alpha}: \alpha \in A\right\}$ generate $I$ and let $x=\Pi x_{\alpha}$ in $M=R^{A}$. By (3) we need only show $I=C(x)$. Since each $x_{\alpha}$ is in the f.g. ideal $I_{i}$ we see from Lemma 5.2 that $x \in M \cdot I_{i}$. Hence $C(x) \subseteq \bigcap I_{i}=I$. Now suppose $J$ is any left ideal of $M$ with $x \in M \cdot J$. Then each $x_{\alpha} \in J$ and so $I \subseteq J$ since the $x_{\alpha}$ 's generate $I$. But $\mathrm{C}(x)$ is the intersection of all such $J$ 's. Thus $I \subseteq C(x)$.

(4) $\Rightarrow(1)$. If $M=\Pi M_{\alpha}$ is a content module then each $M_{\alpha}$ is a content module by Proposition 5.14. Suppose $M=\Pi M_{\alpha}$ where each $M_{\alpha}$ is a content module and $x=\Pi x_{\alpha}$ is in $M$. We need to show $x \in M \cdot C(x)$. By (4) and part (2) of Proposition 5.14, $\mathrm{C}(x)$ is f.g. Thus $x \in M \cdot \mathrm{C}(x)$ iff each $x_{\alpha} \in M_{\alpha} \cdot \mathrm{C}(x)$. Since each $M_{\alpha}$ is a content module, the latter is equivalent to each $C\left(x_{\alpha}\right) \subseteq$ $C(x)$. If $J$ is any left ideal with $x \in M \cdot J$ then clearly each $x_{\alpha} \in M_{\alpha} \cdot J$ and so $C\left(x_{\alpha}\right) \subseteq J$. Since $C(x)$ is the intersection of all these $J$ we thus have $C\left(x_{\alpha}\right) \subseteq$ $C(x)$ as we needed.

We will call a module $M$ an $F C$ module if it is flat and is a content module. $R$ is right $\Pi-F C$ if each $R^{A}$ is a right FC module. $R$ is right $\Pi-C$ if it satisfies the conditions of Theorem 5.15.

By Propositions 2.4 and 5.14, UTL $\Rightarrow$ FC and hence right $\Pi-T \Rightarrow$ right $\Pi-F C$. However Theorems 5.10 and 5.15 show that the latter implication is equivalent to Theorem 5.13. Of course the converse of Theorem 5.13 would be true if FC $\Rightarrow$ UTL. However Example 5.4 in [11] attributed by $\mathrm{Ohm}$ and Rush to Heinzer is of a f.g. FC module $M$ which is not projective and hence not UTL. Since the module $M$ is a pure submodule of $R^{A}$ one may ask if the properties of $M$ are inherited from $R^{A}$, in other words is their ring $R$ a $\Pi$-FC ring and not a $\Pi-T$ ring? The answer is NO.

Proposition 5.16. Let $k$ be any ring. The ring $R$ of eventually constant $k$-sequences is not right $\Pi-C$.

Proof. We consider a sequence a function on the set $N$ of natural numbers. For $n \in N$ we let $f_{n}: N \rightarrow k$ be the function that is zero on $n$ and one elsewhere. Let $I=\bigcap R f_{2 n}$. Note $g \in I$ iff $g(2 n)=0$ for each $n$. Thus each such $g$ must be eventually zero. Hence $I=\Sigma R\left(1-f_{2 n+1}\right)$ and cannot be f.g.

Although we do not know whether $(\Pi-C+\Pi-F) \Rightarrow \Pi-T$ is true, it is easy to see that $\Pi-C$ and $\Pi-F$ are independent of each other. Example 5.1 shows $\Pi-C \neq \Pi-F$ and Example 5.2 shows $\Pi-F \neq \Pi-C$. 
6. Direct products of rings. In this section we wish to determine when a direct product of rings is right II- $X$ where $X$ is the flat, content, flat content, or projective property.

It is well known that the flatness of $R^{A}$ is related to certain left ideals being finitely generated and finitely related. So we start by studying these concepts.

We will call a left module $M$ finitely related (f.r.) if there is an exact sequence $0 \rightarrow K \rightarrow P \rightarrow M \rightarrow 0$ with $P$ projective and $K$ f.g. (Note we do not demand $P$ be f.g.) If both $P$ and $K$ are f.g. we say $M$ is finitely presented (f.p.) and call the exact sequence $a$ finite presentation for $M$.

Suppose $M$ is any left $R$-module. Recall $\mu(M) \leqslant n$ if $M$ is generated by $n$ elements. We also define

$$
\mu^{*}(M)=\sup \{\mu(N): N \text { is a f.g. submodule of } M\} .
$$

The following is a modification of Schanuel's lemma.

Lemma 6.1. Suppose (*) is an exact diagram of left $R$-modules and $P$ is projective.

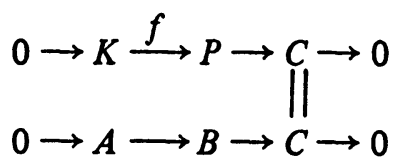

Then

(i) There is an exact sequence $0 \rightarrow K \rightarrow A \oplus P \rightarrow B \rightarrow 0$.

(ii) If $B$ is f.g. and $C$ f.r., then $A$ is f.g.

(iii) If $C$ is f.g. and f.r., then it if f.p.

(iv) If $B$ is f.p. and $A$ f.g., then $C$ is f.p.

Proof. (i). Since $P$ is projective there are maps $h: P \rightarrow B$ and $j: K \rightarrow A$ rendering (*) commutative. It is simple to verify that the maps

$$
\begin{aligned}
k & \mapsto(j(k),-f(k)) & & \text { for } k \in K, \\
(a, p) & \mapsto j(a)+h(p) & & \text { for } a \in A, p \in P,
\end{aligned}
$$

yield the desired exact sequence.

(ii). Since $C$ is f.r. we can choose $P$ and $K$ with $K$ f.g. Then $\mu(A) \leqslant$ $\mu(A \oplus P) \leqslant \mu(K)+\mu(B)<\infty$.

(iii). Since $C$ is f.g. we can choose $B$ to be f.g. projective. Then $C$ is f.p. since by (ii) $A$ is f.g.

(iv). We may assume there is an epimorphism $g: P \rightarrow B$ rendering (*) commutative with $P$ and $L=\operatorname{Ker} g$ both f.g. Since $A$ is f.g. there is a f.g. submodule $Q$ of $P$ with $g(Q)=A$. Then $K=L+Q$ is f.g. and so $C$ is f.p.

Proposition 6.2. For any ring $R$ and any integer $n$ the following condi- 
tions are equivalent:

(i) $R$ is left coherent and $\mu^{*}(R) \leqslant n$.

(ii) $R$ is left coherent and $\mu^{*}(M) \leqslant n \mu(M)$ for each left module $M$.

(iii) For each f.g. left ideal of $R$ there is an exact sequence $R^{n^{2}} \rightarrow R^{n} \rightarrow$ $I \rightarrow 0$.

Proof. (i) $\Rightarrow$ (ii). We claim it suffices to consider $M$ f.g. free and by induction we can assume $M=R^{r} \oplus R$ and $\mu^{*}\left(R^{r}\right) \leqslant n r$. For each f.g. submodule $A$ of $M$, the decomposition of $M$ induces an exact sequence $0 \rightarrow R^{r} \cap A \rightarrow A$ $\rightarrow I \rightarrow 0$ where $I$ is an ideal of $R$. Since $A$ is f.g. so is $I$ and hence $\mu(I) \leqslant n$. Since $R$ is left coherent, $I$ is f.p. and so $R^{r} \cap A$ is f.g. by Schanuel's lemma. Thus $\mu\left(R^{r} \cap A\right) \leqslant n r$ and so $\mu(A) \leqslant n r+n$.

(ii) $\Rightarrow$ (iii). Since $\mu^{*}(R) \leqslant n$ for each f.g. left ideal $I$ of $R$ there is an exact sequence $0 \rightarrow K \rightarrow R^{n} \rightarrow I \rightarrow 0$. Since $R$ is left coherent, $K$ is f.g. and thus $\mu(K) \leqslant n \cdot \mu\left(R^{n}\right) \leqslant n \cdot n$. Thus we can modify the above exact sequence to obtain the desired one.

(iii) $\Rightarrow$ (i). Trivial.

We will call $R$ left $n$-coherent if it satisfies the conditions of Proposition 6.2.

EXAMPLE 6.1. A valuation ring is always 1-coherent, but need not be Noetherian or even $\Pi-T$.

EXAMPLE 6.2. If $k$ is a field, and $n$ a positive integer then the subring

$$
R_{n}=\left\{a+x^{n} f(x): a \in k, f(x) \in k[[x]]\right\}
$$

is a commutative, local, Noetherian $n$-coherent domain which is not $(n-1)$-coherent.

EXAMPLE 6.3. Let $R$ be the trivial ring extension of the integers by the abelian group of rational numbers. Then $\mu^{*}(R) \leqslant 1$, but $R$ is not coherent.

Proposition 6.3. The ring $R=\Pi R_{i}$ is left $n$-coherent iff each $R_{i}$ is left n-coherent.

Proof. It is easily checked that condition (iii) of Proposition 6.2 is valid for $R$ iff it is valid for each $R_{i}$.

If $R$ is a ring and $n$ a positive integer, let

$$
\varphi(n, R)=\sup \left\{\mu(\operatorname{Ker} u): u \in\left(R^{n}\right)^{*}\right\} .
$$

Soublin [13] calls $R$ left uniformly coherent if $\varphi(n, R)<\infty$ for each $n$.

THEOREM 6.4. The ring $R=\Pi R_{i}$ is left coherent iff each $R_{i}$ is left coherent and there is a sequence $0 \leqslant a_{n}<\infty$ with each $B_{n}=\left\{i: \varphi\left(n, R_{i}\right)>a_{n}\right\}$ finite.

Proof. $\Leftrightarrow)$. It is easy to see that each $R_{i}$ is left coherent. If there is no such sequence, then there is an integer $n$ and a sequence $i_{1}, i_{2}, \ldots$ of distinct 
indices such that for each $p>0$ there is $u_{i_{p}} \in\left(R_{i_{p}}^{n}\right)^{*}$ with $\mu\left(\operatorname{Ker} u_{i_{p}}\right)>p$. For $i \notin\left\{i_{p}\right\}$ let $u_{i}=0$ in $\left(R_{i}^{n}\right)^{*}$. Let $u=\Pi u_{i} \in \Pi\left(R_{i}^{n}\right)^{*}=\left(R^{n}\right)^{*}$. Then $\operatorname{Ker} u$ is infinitely generated and hence the image of $u$ is a f.g. left ideal which is not f.r. By Theorem 5.10, $R$ would not be left coherent.

$\Leftrightarrow)$. Let $I$ be a left ideal of $R$ with $\mu(I)=n<\infty$. Let $u \in\left(R^{n}\right)^{*}$ with image $I$. Clearly $u$ can be written

$$
u=\prod_{u_{i}} \in \Pi\left(R_{i}^{n}\right)^{*}
$$

$\operatorname{Ker} u_{i}$ is f.g. since $R_{i}$ is left coherent. Let

$$
r=\max \left\{\mu\left(\operatorname{Ker} u_{i}\right): i \in B_{n}\right\} \text {. }
$$

Clearly $\mu(\operatorname{Ker} u) \leqslant \max \left(a_{n}, r\right)$ and so $I$ is f.p.

COROLlARY (see [13]). The arbitrary product of $R$ with itself is left coherent iff $R$ is left uniformly coherent.

REMARK. Soublin [13] shows that the polynomial ring in two variables over a field is uniformly coherent. Clearly, this ring is not $n$-coherent for any $n$. In general the product of infinitely many uniformly coherent rings is not even coherent.

We now record the following fact needed in the next few theorems. We omit its very simple proof.

LEMmA 6.5. For any collection $\left\{R_{i}\right\}$ of rings, (i) is equivalent to (ii).

(i) If for each $i, J_{i}$ is a f.g. left ideal then $\Pi J_{i}$ is f.g. in $\Pi R_{i}$.

(ii) For some $n<\infty, A_{n}=\left\{i: \mu^{*}\left(R_{i}\right)>n\right\}$ is finite.

THEOREM 6.6. The ring $R=\Pi R_{i}$ is right $\Pi-C$ iff each $R_{i}$ is right $\Pi-C$ and for some $n$ the set $A_{n}=\left\{i: \mu^{*}\left(R_{i}\right)>n\right\}$ is finite.

Proof. $(\Rightarrow)$. It is easy to see that each $R_{i}$ is right $\Pi-C$ if $R$ is. Suppose $\left\{J_{i}\right\}$ is a collection of f.g. left $R_{i}$-ideals. Then each $I_{i}=J_{i} \times \Pi_{j \neq i} R_{j}$ is a f.g. $R$-ideal and hence $I=\bigcap_{i}$ is f.g. But $I=\Pi J_{i}$ and hence some $A_{n}$ is finite by the last lemma.

$\Leftrightarrow)$. By Theorem 5.15, we need only show $\bigcap_{\alpha}$ is f.g. if each $J_{\alpha}$ is a f.g. left ideal of $R$. But since $J_{\alpha}$ is f.g. it has the from $\Pi_{i} J_{\alpha, i}$ where $J_{\alpha, i}$ is f.g. in $R_{i}$. Thus $\bigcap J_{\alpha}=\Pi J_{i}$ where each $J_{i}=\bigcap_{\alpha} J_{\alpha, i}$ is f.g. since $R_{i}$ is right ח-C. Hence by Lemma $6.5, \cap_{\alpha}$ is f.g.

THEOREM 6.7. The ring $R=\Pi R_{i}$ is right $\Pi-F C$ iff each $R_{i}$ is right $\Pi-F C$ and for some $n, A_{n}=\left\{i: \mu^{*}\left(R_{i}\right)>n\right\}$ is finite.

Proof. $(\Rightarrow)$. A direct consequence of Theorems 6.4 and 6.6.

$\Leftrightarrow$ ( $R$ is right $\Pi-C$ by Theorem 6.6. Since each $R_{i}$ is coherent then by Proposition 6.2, $\left\{i: \varphi\left(n, R_{i}\right)>n^{2}\right\} \subseteq A_{n}$. Hence since each $R_{i}$ is right $\Pi-F$, by Theorem 6.4 so is $R$. 
THEOREM 6.8. The ring $R=\Pi R_{i}$ is right $\Pi-T$ iff each $R_{i}$ is right $\Pi-T$ and for some $n$ the set $A_{n}=\left\{i: \mu^{*}\left(R_{i}\right)>n\right\}$ is finite.

Proof. $(\Rightarrow)$. For each set $A, R_{i}^{A}$ is UTL as a right $R$-module since it is a direct summand of $R^{A}$. Hence it is UTL over $R_{i}$ and so $R_{i}$ is right $\Pi-T . A_{n}$ is finite by Theorem 6.6 .

$(\Leftrightarrow)$. Let $x$ be in the right $R$ module $M=R^{A}$. It is easy to see $M=\Pi M_{i}$ with $M_{i}=R_{i}^{A}$ and if $x=\Pi x_{i}$ then $M^{*}(x)=\Pi M_{i}^{*}\left(x_{i}\right)$. Each $M_{i}^{*}\left(x_{i}\right)$ is a f.g. left ideal of $R_{i}$ since $R_{i}$ is right $\Pi-T$. Hence $M^{*}(x)$ is f.g. since $A_{n}$ is finite.

EXAMPLE 6.4. If $k$ is a Dedekind domain and $A$ an infinite set, then $R=$ $k^{A}$ is a commutative $\Pi-T$ ring which is not Noetherian.

The following is a generalization of Corollary 5.2 of [11].

COROLLARY. If $R=\Pi R_{i}$ where each $R_{i}$ is a field, then each content module $M$ over $R$ is a trace module.

Proof. For each nonzero $x$ in $M$ by Corollary 4.4 of [11] there is an idempotent $e_{i}$ with $x e_{i} \neq 0$, and $R_{i}=R e_{i}$ being a field. Clearly, there is a map $u_{i}: M e_{i} \rightarrow R_{i}$ with $u_{i}\left(x e_{i}\right) \neq 0$. Thus $M^{*}(x) \neq 0$ and so $M$ is torsionless. Since $R$ is absolutely flat each $R$-module is flat and hence every submodule is pure. Hence $M$ is a pure submodule of $R^{M^{*}}$. By Theorem $6.8 R$ is $\Pi-T$ and so $R^{M^{*}}$ is UTL. Thus $M$ is UTL.

REMARK. The above Corollary uses the fact that $R$ is absolutely flat, is $\Pi-T$ and each ideal contains a minimal ideal.

We close this section with

THEOREM 6.9. The ring $R=\Pi R_{i}$ is right $\Pi-P$ iff each $R_{i}$ is right $\Pi-P$ and the set $\left\{i: R_{i} \neq 0\right\}$ is finite.

Proof. By Theorem 5.12, $R$ is right $\Pi-P$ iff it satisfies the descending chain condition on f.g. left ideals and is left coherent. Clearly, $R$ satisfies the chain condition iff each $R_{i}$ does and there are only finitely many nonzero $R_{i}$ 's. It is easily seen that in this situation $R$ is left coherent iff each $R_{i}$ is.

Appendix. When is $\Gamma$ always monic or epic?

Recall that if $\Gamma(M, A): M \otimes A \rightarrow \operatorname{Hom}\left(M^{*}, A\right)$ is the canonical map, then $M$ is called UTL if $\Gamma(M, A)$ is monic for all $A$. In this appendix we show that (at least over the integers) no other new class of modules is characterized by fixing one of the variables and requiring $\Gamma$ to be always monic or always epic.

Proposition A.l. $\Gamma(M, A)$ is epic for all $A$ iff $M=P \oplus K$ with $P$ f.g. projective and $K^{*}=0$.

Proof. $(\Rightarrow)$. The natural map $M^{* *} \otimes M^{*} \rightarrow \operatorname{Hom}\left(M^{*}, M^{*}\right)$ is epic since $\Gamma\left(M, M^{*}\right)$ is epic. Therefore $M^{*}$ has a finite projective basis $[5, \mathrm{p} .132]$ and hence is f.g. projective. Thus $M^{* *}$ is also f.g. projective and since $\Gamma(M, R)$ is 
epic, $M \cong M^{* *} \oplus K$ where $K$ is the kernel of $\Gamma(M, R)$. It is easy to see $K^{*}=0$.

$(\Leftrightarrow)$. This second implication is essentially equivalent to the following well known result.

Corollary. $\Gamma(M, A)$ is an isomorphism for all $A$ iff $M$ is f.g. projective.

If $R$ is a field, then each $\Gamma(M, A)$ is monic since $M$ is projective and hence UTL. The converse is essentially true.

Proposition A.2. For any ring $R$ the following are equivalent.

(i) $R$ is semisimple with minimal conditions.

(ii) Each right module $M$ is UTL.

(iii) $\Gamma(M, A)$ is monic for all cyclic $M$ and $A$.

Proof. (i) $\Rightarrow$ (ii) and (ii) $\Rightarrow$ (iii) are obvious. If (iii) holds then for each right ideal $I$ of $R$, the module $M=R / I$ is UTL by (iii) $\Rightarrow$ (i) of Theorem 3.2. Hence $M$ is projective and so $I$ is a direct summand of $R$.

The opposite type of situation holds when $R$ is the ring $\mathbf{Z}$ of rational integers which we assume it to be from here on.

For the rest of this section $P$ is the direct product of a countable number of copies of $Z$ and $S$ is the subgroup consisting of the corresponding direct sum of copies of $\mathbf{Z}$.

Proposition A.3. If $M=n P+S$ where $n$ is a nonzero integer, there is an exact sequence

$$
0 \rightarrow P / M \rightarrow M \otimes \mathrm{Z} / n \mathrm{Z} \stackrel{\Gamma}{\longrightarrow} \operatorname{Hom}\left(M^{*}, \mathrm{Z} / n \mathrm{Z}\right) \rightarrow P / M \rightarrow 0 .
$$

Proof. We start by showing the restriction $P^{*} \rightarrow M^{*}$ is an isomorphism. Since $n P \subseteq M$ it is easy to see $n M^{*} \subseteq P^{*} \subseteq M^{*}$. It is known that $S$ is reflexive $\left[7\right.$, p. 106] and since $P \cong S^{*}$ it is easy to see that $\left\{u_{i} ; e_{i}^{* *}\right\}$ is a projective basis for $P^{*}$ where $u_{i} \in P^{*}$ is the $i$ th projection map and $e_{i}^{* *}$ is the evaluation at the $i$ th standard basis of $S$. Since $\left(P^{*}\right)_{*}=M^{*}$ and each $e_{i}^{* *} \in M^{* *}$ (since $S \subseteq M$ ), it is easily seen that $\left\{u_{i}, e_{i}^{* *}\right\}$ is a projective basis for $M^{*}$ also. Hence $M^{*}=P^{*}$.

Since $M^{*} \cong S$ is projective we have an exact commutative diagram

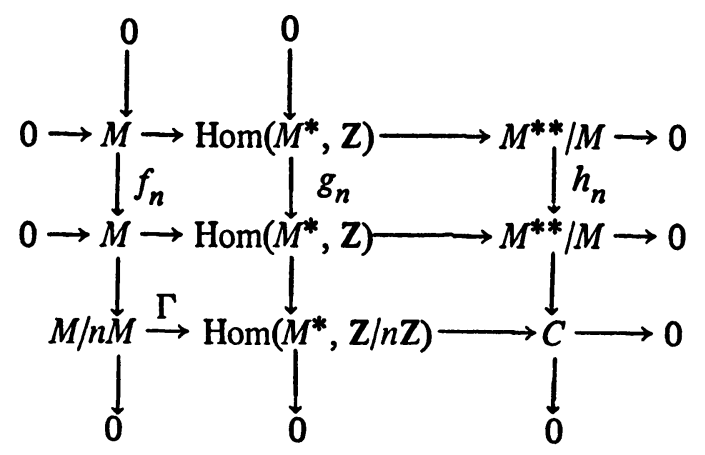


where $f_{n}, g_{n}, h_{n}$ are all multiplication by $n$ and the other maps are natural. Using the identifications $M^{* *}=P^{* *}=P$ we see $M^{* *} / M=P / M$ and $h_{n}=0$. Hence $C=P / M$ and $\operatorname{Ker} \Gamma=\operatorname{Ker} h_{n}=P / M$.

Corollary 1. An abelian group $A=0$ if $\Gamma(M, A)$ is monic for all $M$.

Proof. Since $\mathrm{Q}^{*}=0$ we see $\mathrm{Q} \otimes A=0$ and thus $A$ is a torsion group. If $A \neq 0$ then there is $n \neq 0$ and a monomorphism $\mathrm{Z} / n \mathrm{Z} \rightarrow A$. Let $M=n P$ $+S$. Since $M$ is torsion free, $M \otimes \mathrm{Z} / n \mathrm{Z} \rightarrow M \otimes A$ is monic. Hence if $\Gamma(M, A)$ is monic, also $\Gamma(M, Z / n Z)$ is monic. By Proposition A.3 this is false.

Corollary 2. An abelian group $A=0$ if $\Gamma(M, A)$ is epic for all $M$.

Proof. Since $\Gamma(P, A)$ is epic, each $f: P^{*} \rightarrow A$ has finitely generated image. Since $P^{*} \cong S$ is countable free abelian, $A$ is finitely generated. Hence if $A \neq 0$, there is an epic $A \rightarrow Z / n Z$ for some $n \neq 0$. Let $M=n P+S$. Since $\Gamma(M, A)$ and $\operatorname{Hom}\left(M^{*}, A\right) \rightarrow \operatorname{Hom}\left(M^{*}, \mathrm{Z} / n \mathbf{Z}\right)$ are epic, so is $\Gamma(M, \mathbf{Z} / n \mathbf{Z})$. By Proposition A.3 this is false.

\section{REFERENCES}

1. F. Albrecht, On projective modules over semi-hereditary rings, Proc. Amer. Math. Soc. 12 (1961), 638-639. MR 23 \#A3766. 68-122.

2. R. Baer, Abelian groups without elements of finite order, Duke Math. J. 3 (1937),

3. H. Bass, Projective modules over free groups are free, J. Algebra 1 (1964), 367-373. MR 31 \#2290.

4. N. Bourbaki, Eléments de mathématique. Fasc. XXVII. Algèbre commutative. Chap. 1. Modules plats, Actualités Sci. Indust., no. 1290, Hermann, Paris, 1961. MR 36 \#146.

5. H. Cartan and S. Eilenberg, Homological algebra, Princeton Univ. Press, Princeton, N. J., 1956. MR 17, 1040.

6. S. U. Chase, Direct products of modules, Trans. Amer. Math. Soc. 97 (1960), 457473. MR 22 \#11017.

7. P. A. Griffith, Infinite Abelian group theory, Univ. of Chicago Press, Chicago, Ill. and London, 1970. MR 44 \#6826.

8. L. Gruson and M. Raynaud, Critères de platitude et de projectivité. Techniques de "platification" d'un module, Invent. Math. 13 (1971), 1-89. MR 46 \#7219.

9. I. Kaplansky, Projective modules, Ann. of Math. (2) 68 (1958), 372-377. MR 20 \#6453.

10. J. Ohm and D. E. Rush, Finiteness of $I$ when $R[X] / I$ is flat, Trans. Amer. Math. Soc. 171 (1972), 377-408. MR 46 \#5303.

11. Content modules and algebras, Math. Scand. 31 (1972), 49-68. \#6473.

12. B. T. Stenström, Pure submodules, Ark. Mat. 7 (1967), 159-171. MR 36

13. J.-P. Soublin, Anneaux et modules cohérents, J. Algebra 15 (1970), 455-472. MR 41 \#5422.

DEPARTMENT OF MATHEMATICS, OAKLAND UNIVERSITY, ROCHESTER, MICHIGAN 48063

Current address: Department of Mathematical Sciences, New Mexico State University, Las Cruces, New Mexico 88003 